\title{
Etanol como aditivo para combustibles: estudio de la oxidación a alta presión de sus mezclas con acetileno
}

\author{
Lorena Marrodán, Miguel Fuster, Ángela Millera, Rafael Bilbao, María U. Alzueta \\ Grupo de Procesos Termoquímicos (GPT) \\ Instituto de Investigación en Ingeniería de Aragón (I3A) \\ Universidad de Zaragoza, Mariano Esquillor s/n, 50018, Zaragoza, Spain. \\ Tel. +34-876555451, e-mail: marrodan@unizar.es
}

\begin{abstract}
Se ha evaluado el comportamiento del etanol como aditivo a través del estudio experimental y de modelado cinético de la oxidación a alta presión (10 y 40 bar) de sus mezclas con acetileno para diferentes condiciones de temperatura, relaciones aire/combustible y cantidades de etanol añadidas a la mezcla reaccionante.
\end{abstract}

\section{Introducción y objetivos}

Entre las diferentes estrategias para la minimización de emisiones contaminantes producidas en los motores diésel, la refomulación de los combustibles tradicionales es una de las alternativas más atractivas ya que sus efectos son inmediatos $y$, además, se puede implementar sin necesidad de rediseñar los equipos. Esta reformulación consiste en la sustitución total o parcial del combustible por combustibles alternativos que puedan ser obtenidos a partir de biomasa mediante procesos de biorefinería. Actualmente, el biodiésel es un combustible ampliamente utilizado y compuestos orgánicos oxigenados, como el etanol y el metanol, ya se emplean en los motores de gasolina. En la bibliografía se pueden encontrar diversos estudios referentes a mezclas de combustibles (o compuestos modelo representativos de los mismos) y compuestos oxigenados llevados a cabo en diferentes sistemas de combustión, desde llamas a motores (e.g. [1]).

Hasta el momento, el etanol es uno de los alcoholes más estudiados y su utilización, directamente o como aditivo en los motores de gasolina, está muy extendida. Sin embargo, si se compara con el diésel, el etanol presenta un menor índice de cetano, flash point y poder calorífico, por lo que no puede usarse directamente en los motores diésel. Para superar estos inconvenientes, debe ser mezclado con diésel o biodiésel, y muchos autores indican, que trabajando en las condiciones adecuadas, se consiguen reducir las emisiones de CO, materia particulada (tipo hollín) y óxidos de nitrógeno [2].
Por lo tanto, resulta interesante llevar a cabo estudios que nos ayuden a entender de qué manera se consiguen reducir estas emisiones. Por ejemplo, mediante el análisis de los efectos de añadir etanol en el comportamiento de los precursores de hollín (hidrocarburos pequeños o compuestos aromáticos).

En este contexto, y teniendo en cuenta la escasez de trabajos en reactores de flujo a alta presiones disponibles en la bibliografía, a pesar de la gran relevancia de estos estudios para su aplicabilidad a motores de combustión interna, en el presente trabajo se ha realizado un estudio experimental y de modelado cinético de la oxidación a alta presión de mezclas $\mathrm{C}_{2} \mathrm{H}_{2}$-etanol. Los resultados experimentales obtenidos han sido empleados para validar un mecanismo cinético-químico, en el que el grupo de investigación lleva años trabajando, y que es capaz de describir la oxidación de ambos compuestos y de sus mezclas en las condiciones estudiadas. Ello permitirá extender la aplicabilidad del modelo a otras condiciones de operación y ser utilizado como mecanismo de predicción.

\section{Metodología}

La parte experimental de este trabajo, realizada en una instalación de fase gas a alta presión (descripción más detallada en [3]), incluye el estudio de la oxidación de mezclas $\mathrm{C}_{2} \mathrm{H}_{2}$ (500 ppm) y etanol (50-200 ppm). En los experimentos se ha variado la concentración de oxígeno en la mezcla reaccionante, desde condiciones reductoras hasta oxidantes (modificando para ello el valor de $\lambda$, definida como la relación entre el oxígeno real alimentado y el oxígeno estequiométrico). Los reactantes se diluyen en $\mathrm{N}_{2}$ para minimizar los efectos térmicos de las reacciones, de manera que el flujo total de gas alimentado es de $1000 \mathrm{mLN} / \mathrm{min}$ (trabajando con un tiempo de residencia: $\left.\mathrm{t}_{\mathrm{r}}[\mathrm{s}]=261 * \mathrm{P}[\mathrm{bar}] / \mathrm{T}[\mathrm{K}]\right)$. Para evaluar la influencia de la presión sobre el proceso de oxidación, se han probado dos presiones diferentes, 10 y 40 bar, 
mientras que la influencia de la temperatura se ha analizado en el intervalo 575-1075 K.

Por otro lado, los cálculos teóricos se han realizado utilizando el software CHEMKIN-PRO, introduciendo como ficheros de entrada el mecanismo cinético (791 reacciones y 137 especies) y los datos termodinámicos correspondientes a las especies involucradas.

\section{Resultados}

En la Figura 1, se comparan los resultados experimentales (símbolos) y teóricos (líneas) correspondientes a la conversión de $\mathrm{C}_{2} \mathrm{H}_{2}$ y formación de $\mathrm{CO}$ (como ejemplo de los productos obtenidos) para una presión de 10 bar, cuando se han añadido únicamente $50 \mathrm{ppm}$ de etanol y para diferentes concentraciones de oxígeno (distintos valores de $\lambda$ ). No se observa una clara influencia de la concentración de oxígeno. La oxidación de $\mathrm{C}_{2} \mathrm{H}_{2}$ y la formación de $\mathrm{CO}$ se inician aproximadamente a las mismas temperaturas independientemente del valor de $\lambda$. Tampoco se observa una influencia clara en la conversión de etanol o en la formación del resto de los productos cuantificados experimentalmente (resultados no mostrados). La cantidad de etanol añadida a la mezcla, como se puede observar en la Figura 2, tampoco modifica el régimen del oxidación del $\mathrm{C}_{2} \mathrm{H}_{2}$ bajo las condiciones estudiadas en el presente trabajo. Únicamente un aumento de la presión, cuando se pasa de 10 a 40 bar, provoca un desplazamiento a menores temperaturas de la temperatura a la que se inicia la conversión de $\mathrm{C}_{2} \mathrm{H}_{2}$, produciéndose a unos $750 \mathrm{~K}$. Esta temperatura es ligeramente superior a la que comienza la conversión de $\mathrm{C}_{2} \mathrm{H}_{2}$, unos $725 \mathrm{~K}$, cuando se analiza su oxidación sin presencia de etanol en unas condiciones de estudio similares, pero a una presión de 60 bar [4].

\section{Conclusiones y trabajo futuro}

A la vista de los resultados obtenidos, ni la concentración de oxígeno, ni la cantidad de etanol añadida a la mezcla reaccionante tienen una clara influencia en la conversión de $\mathrm{C}_{2} \mathrm{H}_{2}$. Únicamente un aumento de la presión desplaza dicha conversión hacia menores temperaturas, por lo que aparentemente no existe una interacción entre ambos compuestos analizados. Dadas las buenas predicciones del modelo, se profundizará en el estudio de la interacción o no de dichos compuestos a través de la identificación de los principales caminos de reacción determinados con ayuda del mecanismo cinético.

\section{Agradecimientos}

Los autores quieren expresar su gratitud al Gobierno de Aragón y Fondo Social Europeo y a MINECO y FEDER (Proyecto CTQ2015-65226) por la financiación recibida. Lorena Marrodán agradece al Gobierno de Aragón la beca predoctoral concedida.

\section{REFERENCIAS}

[1]. SARATHY, S.M., OßWALD, P., HANSEN, N. and KOHSE-HÖINGHAUS, K. Alcohol combustion chemistry. Progress in Energy and Combustion Science. 2014, 44, 40-102.

[2]. AN, H. and YANG, J.L. Effects of ethanol addition on biodiesel combustion: A modeling study. Applied Energy. 2015, 143, 176-188.

[3]. MARRODÁN, L., MILLERA, Á., BILBAO, R. and ALZUETA, M.U. High-pressure study of methyl formate oxidation and its interaction with NO. Energy and Fuels. 2014, 28(9), 6107-6115.

[4]. GIMÉNEZ-LÓPEZ, J., RASMUSSEN, C.T., HASHEMI, H., GAO, Y., MARSHALL, P., GOLDSMITH, F. and GLARBORG, P. Experimental and kinetic modeling study of $\mathrm{C}_{2} \mathrm{H}_{2}$ oxidation at high pressure. International Journal of Chemical Kinetics. 2016, 48(11), 724-738.

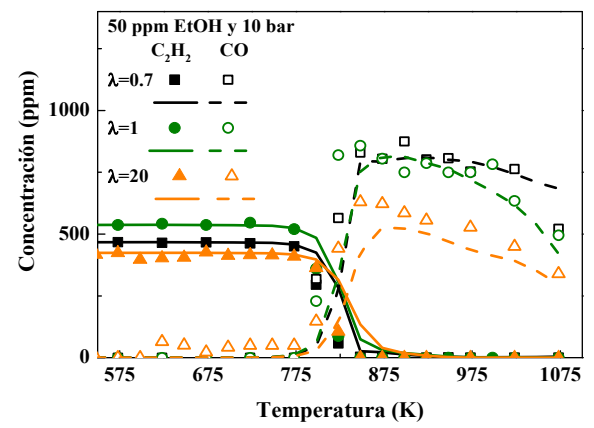

Fig. 1. Influencia de la temperatura y concentración de oxígeno ( $\lambda$ ) en el consumo de $\mathrm{C}_{2} \mathrm{H}_{2}$ y formación de $\mathrm{CO}$.

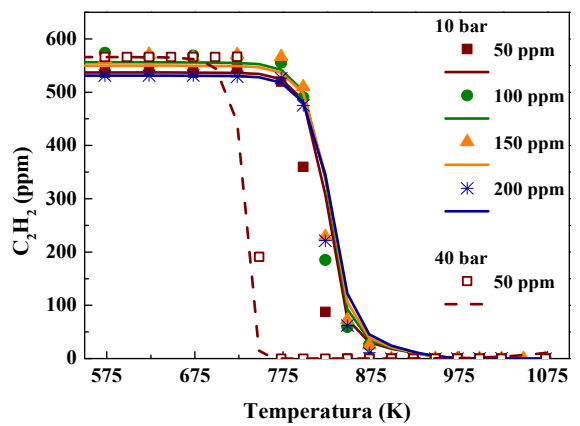

Fig. 2. Influencia de la cantidad de etanol añadida, de la presión (10 y 40 bar) y de la temperatura para condiciones estequiométricas $(\lambda=1)$. 